\title{
Referring Pathologist
}

National Cancer Institute

\section{Source}

National Cancer Institute. Referring Pathologist. NCI Thesaurus. Code C51859.

A pathologist that directs a patient to an additional source of medical expertise for assistance, examination, information, or treatment. 